\title{
Modelos epidemiológicos y la pandemia COVID-19 en Chile
}

\author{
Epidemiological models and the COVID-19 pandemic in Chile
}

\author{
Mauricio Canals $\mathrm{L}^{1}$
}

Palabras clave: COVID-19, Chile, epidemiología, modelos, número reproductivo.

"Recién estamos comenzando a conocer algo, cuando somos capaces de cuantificarlo" (William Thompson, Lord Kelvin, 1824-1907)

El aumento de la población, la globalización y el cambio climático han significado un cambio en la prevalencia y aparición de nuevas enfermedades infecciosas, muchas de ellas zoonóticas y transmitidas por insectos hematófagos, con cambios en los patrones espaciales y temporales de reservorios, vectores y hospederos. Esto ha significado la emergencia y re-emergencia de numerosas enfermedades. Más del 70\% de estas son zoonóticas, donde el factor más importante es la transgresión de la barrera entre especies, concepto conocido como "spillover" (Jones, 2008). Últimamente, han llamado la atención las enfermedades cuyo reservorio son los murciélagos, principalmente los frugívoros. Los murciélagos tienen al menos 76 virus de peligro potencial para el humano, entre los que destacan los virus Rabia, Marburg, Hendra, Nipah, Menangle, Tioman, Ébola y los coronavirus SARS y MERS (Callisher, 2006). Los estudios actuales muestran casi con seguridad que el virus SARS CoV-2 tiene un origen en murciélagos frugívoros (Andersen, 2020; Lam, 2020). Este nuevo "spillover" está impactando la humanidad con una gran pandemia (COVID-19) y actualmente ya cuenta con cerca de 8,5 millones de casos reportados y cerca de 460 mil fallecidos afectando todos los aspectos de la vida de la humanidad (WHO, 2019).

Una pandemia de la magnitud que ha tenido ésta, ha puesto a toda la comunidad científica mundial a trabajar en forma acelerada en todos los aspectos relacionados con el virus y su propagación. Queremos saber su velocidad de propagación, cuándo será el peak, cuánto durará la pandemia, cuál es la eficacia de las intervenciones epidemiológicas, cuáles son los mejores indicadores para la buena toma de decisiones, qué pasará después, etc. Es en estos aspectos, en los que tiene un rol importante la modelación con una fuerte base teórica sustentada en la epidemiología.
Un ejemplo de esto es la ayuda que ha proporcionado el concepto de $\mathrm{R}_{0}$ en el caso de la pandemia COVID-19 en Chile. Derivado de los modelos epidemiológicos de compartimentos SIR (susceptibles-infectados-removidos), el concepto se concibió inicialmente como una constante característica para la transmisión de una enfermedad infecciosa que representa el número de casos nuevos que se producen en un intervalo serial (intervalo promedio entre contagio y contagio) cuando toda la población es susceptible. Sin embargo, durante las epidemias la fracción de susceptibles es variable $(\mathrm{q}(\mathrm{t}))$ y la transmisión es afectada por las intervenciones epidemiológicas. El número reproductivo efectivo $\operatorname{Re}(\mathrm{t})=\mathrm{q}(\mathrm{t}) \mathrm{R}(\mathrm{t})$. $\mathrm{Si} \mathrm{t}=\mathrm{to} ; \mathrm{q}(\mathrm{t})=1$ y $\mathrm{R}(\mathrm{t})=\mathrm{Re}(\mathrm{t})=\mathrm{R}_{0}($ Canals, 2017). La proporción $\mathrm{q}(\mathrm{t})$ se va reduciendo a lo largo de la epidemia en forma natural por el "efecto rebaño" (Chowell, 2009; Anderson, 2020). Pero a menos que se tenga una vacuna, es un proceso muy largo y necesariamente tendría como consecuencia gran cantidad de fallecidos y saturación de los sistemas de salud. Entonces los modelos matemáticos nos sugieren que hay que acelerar el proceso disminuyendo $\mathrm{R}(\mathrm{t})$.

Los modelos SIR muestran que $\mathrm{R}(\mathrm{t})$ se puede expresar como: $\mathrm{R}(\mathrm{t})=$ $(\beta S o) /(\gamma+\mu)$ donde $\beta$ es el coeficiente de transmisión, $\gamma$ es la tasa de recuperación y $\mu$ la tasa de mortalidad. Es decir, $\mathrm{R}(\mathrm{t})$ es el producto del potencial reproductivo ( $\beta$ So) por la esperanza de vida infectante $(1 /(\gamma+\mu))$. La reducción de $R_{0}$ se puede lograr aumentando la tasa de recuperación, $\gamma$, es decir encontrando un adecuado tratamiento, lo que hasta ahora no ha resultado posible. Entonces el objetivo principal de la mitigación es disminuir el coeficiente de transmisión ( $\beta$ ). Este lo podemos descomponer en: $\beta=b P(I / C) P(C)$, donde $b$ es la tasa de contacto entre las personas, $\mathrm{P}(\mathrm{C})$, la probabilidad de contacto infectante y $\mathrm{P}(\mathrm{I} / \mathrm{C})$ es la probabilidad que un contacto infectante resulte en una infección (Lloyd-Smith, 2009; Anderson, 2020). Las intervenciones epidemiológicas debieran ir dirigidas a la disminución de uno o más de estos tres factores (Canals, 2020).

(1) Programa de Salud Ambiental, ESP y Departamento de Medicina, Facultad de Medicina, Universidad de Chile. 
a) Probabilidad de que un contacto infectante resulte en infección $(\mathrm{P}(\mathrm{I} / \mathrm{C})$ : vacuna, uso de mascarillas, medidas de higiene personal, uso de desinfectantes, lavado de manos, etc.

b) Tasa de contacto(b): medidas de inmovilidad, desagregación y distanciamiento social.

c) Probabilidad de contacto infectante $P(C)$ : trazabilidad y aislamiento de infectados y contactos, cierre de colegios y universidades, cuarentenas, cordones sanitarios y cierre de fronteras.

La dinámica ecológica y evolutiva de las infecciones se desarrolla en una amplia gama de escalas temporales y espaciales, niveles de organización y con diferentes mecanismos de transmisión. Muchos factores, incluido el aumento de la resistencia a los antimicrobianos, el aumento de la conectividad humana y el comportamiento humano cambiante, elevan la prevención y el control desde un nivel local a un asunto de interés internacional. Frente a esta complejidad, los modelos matemáticos ofrecen herramientas valiosas para sintetizar información para comprender los patrones epidemiológicos y para desarrollar evidencia cuantitativa racionalizando la toma de decisiones en salud.

\section{Referencias}

Andersen KG, Rambaut A, Lipkin WI, Holmes EC \& Garry RF. (2020). The proximal origin of SARS-CoV-2. Nature medicine $\mathbf{2 6}, \mathbf{4 5 0 - 4 5 2 .}$

Anderson RM, Heesterbeek H, Klinkenberg D, Hollingsworth TD (2020). How will country-based mitigation measures influence the course of the COVID-19 epidemic? The Lancet 395, 931-934.
Canals M. (2017) Introducción a la epidemiología matemática. Edición Sociedad Chilena de Parasitología, Santiago, Chile.

Canals M. (2020) Conceptos para una Buena toma de decisiones en la pandemia COVID-19 en Chile. Rev Chil Infecto/ 37, 170-172.

Callisher CH, Childs JE, Field HE, Holmes KV \& Schountz T. (2006) Bats: important reservoir hosts of emerging viruses. Clin Microbiol Rev 19, 531-45.

Chowell G, Hyman J M, Bettencourt L M A \& Castillo-Chávez C. (2009) Mathematical and statistical estimation approaches in epidemiology. Heidelberg. Springer.

Jones KE, Patel N G, Levy MA, Storeygard A, Balk D, Gittleman JL \& Daszak, P. (2008). Global trends in emerging infectious diseases. Nature 451, 990-993.

Lam TTY, Shum MH, Zhu HC, Tong YG, Ni XB, Liao YS, et al. (2020) Identifying SARS-CoV-2 related coronaviruses in Malayan pangolins. Nature.

Lloyd-Smith J, George D, KM Pepin, Pitzer VE, Pullian JRC, Dobson $A P$, et al. (2009) Epidemic dynamic at the human-animal interface. Science 326,1362-1367.

WHO Coronavirus disease (COVID-2019) situation reports. Situation report 99.https:// Accedido en :www.who.int/emergencies/ diseases/novelcoronavirus-2019/situation-reports/ el 23 de junio de 2020 . 